\title{
Association of high levels of $\alpha$-defensins and S100A proteins with Candida mannan detection in bronchoalveolar lavage fluid of preterm neonates
}

\author{
Giovanni Vento', Alessandra Lio', Chiara Tirone', Claudia Aurilia', Milena Tana' ', Andrea Piras'1, Cinzia Ricci', Sarah Perelli', \\ Costantino Romagnoli', Brunella Posteraro², Federica lavarone ${ }^{3}$, Tiziana Cabras ${ }^{4}$, Chiara Fanali ${ }^{5}$, Irene Messana ${ }^{4}$ \\ and Massimo Castagnola ${ }^{3}$
}

BACKGROUND: Candida mannan (Mn) detection in bronchoalveolar lavage fluid (BALF) was shown to be useful for earlier identification and preemptive therapy targeting in preterm infants at high risk of invasive Candida infection. We investigated whether early detection of Candida Mn in BALF is associated with the presence of some neutrophilic products, as markers of prenatal infection/inflammation.

METHODS: BALF specimens were collected during the first $48 \mathrm{~h}$ of life from mechanically ventilated preterm newborns. Samples were analyzed by high-performance liquid chromatography-electrospray ionization-mass spectrometry. The relative amounts of $\alpha$-defensins $1-4$ and S100A proteins were measured by extracted ion current peak area. Absolute and differential white cell counts in BALF were obtained. Mn antigen concentrations were determined by the Platelia Candida antigen kit.

RESULTS: Twenty-five studied neonates were divided into two groups: Mn-positive group and Mn-negative group. Levels of $\alpha$-defensins 1-4 and S100A12 were significantly higher in the Mn-positive group than in the Mn-negative group. Moreover, positive significant correlations between the absolute number of neutrophils and the levels of $\alpha$-defensins $1-4$ and S100A8 were observed.

CONCLUSION: The detection of Mn antigen in BALF of preterm infants is consistent with evidence of an innate immune response in their lungs as demonstrated by higher levels of $\alpha$-defensins and S100A proteins.

S ystemic fungal infections are a significant problem in preterm infants in the neonatal intensive care unit. Fungal colonization is considered an important predictor of progression to invasive Candida infection (ICI) $(1,2)$. Very-low-birth-weight neonates with endotracheal Candida colonization were shown to carry an additional risk of developing systemic candidiasis (3). In high-risk patients, early diagnosis of ICI is essential; late treatment of this disease often results in severe complications and high mortality $(4,5)$. In certain high-risk patients, empiric antifungal therapy has been shown to improve outcomes, but the most recent guidelines by the Infectious Diseases Society of America for the management of adult and pediatric patients with invasive and mucosal candidiasis do not address the issue of empiric therapy in infants (6). Nevertheless, a recent retrospective analysis on 136 infants with birth weight $<1,000 \mathrm{~g}$ and age $<120 \mathrm{~d}$ has been conducted comparing infants who received empiric antifungal therapy with those who did not. These authors found no differences in the single outcomes (greater survival, shorter time to clearance of Candida, and lower incidence of neurodevelopmental impairment), but they observed an improved survival without neurodevelopmental impairment in the infants who received empiric antifungal therapy at 18-22 mo of adjusted age (7). Recently, we documented the utility of Candida mannan (Mn) detection in bronchoalveolar lavage fluid (BALF) for earlier identification and preemptive therapy targeting preterm infants at high risk of ICI (8). Mn in BALF was detected in the first days of life in most patients, suggesting that Candida colonization occurs very early in preterm infants. It has never been demonstrated that the presence of Mn causes pulmonary inflammation and/ or stimulates innate host defense of the lung.

Several articles have been published on the proteomic composition of human BALF, especially for adults (9-12). Among detected proteins that are receiving further attention, especially for their function and involvement in acute and chronic inflammatory conditions, are the $\alpha$-defensins and S100A member proteins (calgranulins) $(13,14)$. To date, six $\alpha$-defensins have been characterized: the human neutrophil peptides 1-4, mainly produced by neutrophils, and the human defensins 5-6 expressed by the Paneth cells (15-18). Defensins have a broad spectrum of antimicrobial activity against bacteria, fungi, and even some enveloped viruses. In various adult infectious lung diseases, elevated levels of $\alpha$-defensins in BALF have been

\footnotetext{
'Department of Pediatrics, Division of Neonatology, Università Cattolica del Sacro Cuore, Rome, Italy; ${ }^{2}$ Institute of Hygiene, Università Cattolica del Sacro Cuore, Rome, Italy; ${ }^{3}$ Institute of Biochemistry and Clinical Biochemistry c/o Institute of Chemistry of Molecular Recognition (CNR) and International Scientific Institute (ISI), Paolo VI, Università Cattolica del Sacro Cuore, Rome, Italy; ${ }^{4}$ Department of Life and Environmental Sciences, Università di Cagliari, Monserrato Campus, Monserrato, Cagliari, Italy; ${ }^{5}$ Integrated Research Center, Università Campus-Biomedico, Rome, Italy. Correspondence: Giovanni Vento (vento@rm.unicatt.it) 


\section{Articles | ventoetal.}

reported $(19,20)$, and we recently investigated the presence of $\alpha$-defensins 1-4 in BALFs of premature newborns with and without pneumonia (21): they were more expressed in cases of bacterial and fungal pneumonia, suggesting that they could be involved in innate host defense of the lung against microbial infection in preterm newborns. S100A8 and S100A12, together with S100A9, are members of the subgroup of S100A proteins named calgranulins. They provide anti-infective and anti-inflammatory functions for the mammalian host and are expressed prevalently in the cytosol of neutrophils and monocytes. They adopt several different mechanisms and roles to protect the host. These functions include antimicrobial activity, chemokine-like activities, oxidant scavenging, and leukocyte chemoattractant activity (22).

McMorran et al. (13) conducted a proteome-based analysis of BALFs from young children suffering from cystic fibrosis with and without lung infection. They detected abnormally high concentrations of $\alpha$-defensin 1 and 2, S100A8, S100A9, and S100A12, as well as novel forms of S100A8 and S100A12, in BALFs of early cystic fibrosis lung disease.

Of note, Buhimschi et al. (23) have recently investigated the changes in amniotic fluid (AF) proteome in the context of inflammation and decidual hemorrhage-induced preterm birth. Proteomic mapping of the AF revealed a profile that was highly characteristic of intra-amniotic inflammation: the presence of four neutrophilic products- $\alpha$-defensin $1, \alpha$-defensin 2 , S100A8 protein (calgranulin A), and S100A12 protein (calgranulin C) - was highly predictive of preterm birth, whether inflammation was secondary to infection or bleeding. Taking into account that $90-99 \%$ of polymorphonuclear leukocytes identified in AF during intra-amniotic infection are of fetal origin (24), this proteomic profile reflects the fetal immune response to intra-amniotic infection. Moreover, in subsequent studies, the presence of S100A12 was shown to be strongly associated with funisitis, whereas S100A8 was linked with neonatal sepsis (25).

The aim of this work was to evaluate the levels of $\alpha$-defensins and S100A proteins, as markers of prenatal infection/inflammation, in BALF samples of premature newborns, in relation to the detection of Candida Mn antigen. Specifically, using a proteomic approach, we determined the relative amounts of $\alpha$-defensins $1-4$ and some members of S100A family proteins, such as S100A8, four isoforms of S100A9 (short form, long form, and two of their phosphorylated forms), and S100A12.

\section{RESULTS}

Of 29 eligible patients during the study period, 25 who fulfilled the inclusion criteria were enrolled in the study and 4 were excluded because of the lack of parent's informed consent. A total of 25 BALF specimens were collected. Of the 25 neonates, $18(72 \%)$ had positive Mn test results (Mn-positive group) and 7 (28\%) did not (Mn-negative group). The mean \pm SD times of BALF collection for the Mn-positive and $\mathrm{Mn}$-negative groups were $35.7 \pm 6.9 \mathrm{~h}$ and $36.4 \pm 8.1 \mathrm{~h}$, respectively $(P=0.83)$. The mean \pm SD amount of fluid recovered varied between 75 and $80 \%$ of the initial lavage fluid instilled for all BALF samples
$(1.1 \pm 0.1 \mathrm{ml}$ in the Mn-negative group and $1.0 \pm 0.05 \mathrm{ml}$ in the Mn-positive group, $P=0.25$ ). Table 1 shows the characteristics of patients. There were no statistically significant differences in gestational age (GA) or birth weight between the two groups.

The number of infants who were small for gestational age was higher in the Mn-positive group than that in the Mn-negative group. At the same time, a higher number of babies in the $\mathrm{Mn}$-positive group relative to the ones in the Mn-negative group were born to mothers with premature rupture of membranes, defined as a premature prolonged rupture of the fetal membranes at least $12 \mathrm{~h}$ before the onset of labor. As regards other prenatal risk factors for ICI, such as vaginal delivery and vaginal swabs positive for Candida spp., no significant differences were found between the two groups (Table 1). All studied infants received one dose of fluconazole for antifungal prophylaxis, before BALF sampling.

The major outcomes of studied patients are summarized in Table 2. During hospitalization, neonates of the Mn-positive group underwent a greater duration of mechanical ventilation and $\mathrm{O}_{2}$ therapy; moreover, the incidence of bronchopulmonary dysplasia was higher in this group than in the Mn-negative group. All the neonates of the Mn-negative group survived; by contrast, only $72 \%$ of neonates survived in the Mn-positive group. The incidence of severe intracranial hemorrhage (grade III or IV) was higher in the Mn-negative group as compared with the Mn-positive group, but the difference was not statistically significant (Table 2).

Concerning BALF cytology, both the median (range) absolute leukocyte and neutrophil counts were higher in the $\mathrm{Mn}$-positive group as compared with the $\mathrm{Mn}$-negative group: $1.50(0.26-11.00)$ leukocytes $\times 10^{9} / 1$ and $1.20(0.20-7.70)$ neutrophils $\times 10^{9} / 1$ vs. $1.14(0.35-2.78)$ leukocytes $\times 10^{9} / 1$ and 1.00

Table 1. Patient characteristics of the groups analyzed

\begin{tabular}{lccc}
\hline & $\begin{array}{c}\text { Mn-negative } \\
\text { group }(n=7)\end{array}$ & $\begin{array}{c}\text { Mn-positive } \\
\text { group }(n=18)\end{array}$ & Pvalue \\
\hline GA (wk) & $25.7 \pm 1.4$ & $26.5 \pm 1.0$ & 0.22 \\
BW (g) & $846 \pm 191$ & $732 \pm 176$ & 0.13 \\
Male & $5(71)$ & $11(61)$ & 1 \\
Appropriate for GA & $7(100)$ & $10(55)$ & 0.06 \\
Antenatal steroids & $4(57)$ & $9(50)$ & 1 \\
Vaginal delivery & $1(14)$ & $1(5)$ & 0.49 \\
pPROM & $2(29)$ & $8(44)$ & 0.66 \\
Vaginal swabs positive & $1 / 3(33)$ & $3 / 8(38)$ & 1 \\
for Candida spp. & & & \\
Apgar score at 1 min & $5(2-8)$ & $4(2-8)$ & 0.58 \\
Apgar score at 5 min & $7(7-9)$ & $7(5-8)$ & 0.36
\end{tabular}

Values expressed as mean \pm SD, median (range), or $n(\%)$.

BW, birth weight; GA, gestational age; Mn, Candida mannan; pPROM, premature prolonged rupture of the fetal membranes at least $12 \mathrm{~h}$ before the onset of labor. antenatal steroids were considered a complete course of prenatal betamethasone, defined as two doses administered $>24 \mathrm{~h}$ but not $>7 \mathrm{~d}$ before delivery. ${ }^{\circ}$ The number of maternal vaginal swabs positive for Candida spp. divided by the total number of maternal vaginal swabs performed before delivery is shown. The mothers of 4 patients in $\mathrm{Mn}$-negative group and the mothers of 10 patients in Mn-positive group did not have vaginal swabs. 
Table 2. Clinical and long-term respiratory outcomes

\begin{tabular}{|c|c|c|c|}
\hline & $\begin{array}{l}\text { Mn-negative } \\
\text { group }(n=7)\end{array}$ & $\begin{array}{l}\text { Mn-positive } \\
\text { group }(n=18)\end{array}$ & $\begin{array}{c}P \\
\text { value }\end{array}$ \\
\hline $\begin{array}{l}\mathrm{O}_{2} \text { dependence at } \\
36 \text { wk postmenstrual } \\
\text { age }\end{array}$ & $1(14)$ & $5 / 13(38)$ & 0.35 \\
\hline Survival to discharge & $7(100)$ & $13(72)$ & 0.27 \\
\hline $\begin{array}{l}\text { Mechanical ventilation } \\
\text { (h) }\end{array}$ & $327(79-1568)$ & $567(51-1080)$ & 0.41 \\
\hline $\mathrm{O}_{2}$ therapy $(\mathrm{h})$ & $839(62-4305)$ & $924(51-2885)$ & 0.93 \\
\hline $\begin{array}{l}\text { Number of surfactant } \\
\text { doses }\end{array}$ & $2.0(1.0-3.0)$ & $2.0(1.0-3.0)$ & 0.65 \\
\hline Air leak & $2(28)$ & $2(11)$ & 0.55 \\
\hline Bacteremia & $4(57)$ & $13(72)$ & 0.64 \\
\hline $\mathrm{ICI}$ & 0 & 0 & 1 \\
\hline Bacterial pneumonia & $4(57)$ & $4(22)$ & 0.16 \\
\hline $\begin{array}{l}\text { Intracranial } \\
\text { hemorrhage } \\
\text { (grade III or IV) }\end{array}$ & $3(43)$ & $1(5)$ & 0.05 \\
\hline $\begin{array}{l}\text { Periventricular } \\
\text { leukomalacia }\end{array}$ & $1(14)$ & 0 & 0.28 \\
\hline $\begin{array}{l}\text { Necrotizing } \\
\text { enterocolitis (stage }>2 \text { ) }\end{array}$ & $2(28)$ & $2(12)$ & 0.55 \\
\hline $\begin{array}{l}\text { Ductus arteriosus } \\
\text { surgically ligated }\end{array}$ & $2(28)$ & $6(33)$ & 1 \\
\hline $\begin{array}{l}\text { Retinopathy of } \\
\text { prematurity (stage }>2 \text { ) }\end{array}$ & $4(57)$ & $7(53)$ & 1 \\
\hline $\begin{array}{l}\text { Length of hospital } \\
\text { stay (d) }\end{array}$ & $94(60-169)$ & $73(18-140)$ & 0.47 \\
\hline
\end{tabular}

$(0.20-2.60)$ neutrophils $\times 10^{9} / 1$, respectively. No differences were found in the median (range) absolute number of alveolar macrophages between the two groups: $0.14(0.03-2.40)$ alveolar macrophages $\times 10^{9} / 1$ in the Mn-positive group vs. 0.15 (0.10-0.34) alveolar macrophages $\times 10^{9} / 1$ in the Mn-negative group.

The analysis of $\alpha$-defensins and S100A proteins was performed using a proteomic approach. The list of analyzed proteins together with their molecular weights is given in Table 3. The $\alpha$-defensins were detected in all samples, and their levels were significantly higher in the Mn-positive group as compared with the Mn-negative group (Figure 1).

Significantly higher levels of S100A12 protein were observed in the Mn-positive group than in the Mn-negative group, whereas no differences were observed between the two groups for S100A8 protein (Figure 2). Concerning S100A9 proteins, only the isoform S100A9-long was detected in two samples of the Mn-positive group (data not shown).

We evaluated the presence of a correlation between the absolute number of neutrophils and the corresponding levels of all the $\alpha$-defensins, S100A8, and S100A12 in only 22 BALF samples because of the absence of the cytological data in 3 samples of the Mn-positive group, due to technical
Table 3. Peptides and proteins analyzed in BALF samples together with their experimental molecular weight (MW exp), Swiss-Prot identification code, and posttranslational modifications (PTMs)

\begin{tabular}{|c|c|c|c|}
\hline Protein & $\begin{array}{l}\text { MW exp } \\
(\mathrm{Da})\end{array}$ & $\begin{array}{l}\text { Swiss-Prot } \\
\text { code }\end{array}$ & PTMs \\
\hline$\alpha$-Defensin 1 & $3,442.0$ & P59665 & $3 S-S$ \\
\hline$\alpha$-Defensin 2 & $3,371.0$ & P59665/6 & $3 S-S$ \\
\hline$\alpha$-Defensin 3 & $3,486.1$ & P59666 & $3 S-S$ \\
\hline$\alpha$-Defensin 4 & $3,707.8$ & P12838 & $3 S-S$ \\
\hline S100A8 (calgranulin A) & 10,834 & P05109 & - \\
\hline $\begin{array}{l}\text { S100A9 (calgranulin B) } \\
\text { short }\end{array}$ & 12,689 & P06702 & $\begin{array}{l}\text { Acetyl, MTCKM } \\
\text { missing (N-term) }\end{array}$ \\
\hline $\begin{array}{l}\text { S100A9 (calgranulin B) } \\
\text { short P }\end{array}$ & 12,769 & P06702 & $\begin{array}{l}\text { Acetyl, MTCKM } \\
\text { missing (N-term), } \\
\left.\text { phosph ( } T_{108}\right)\end{array}$ \\
\hline $\begin{array}{l}\text { S100A9 (calgranulin) } \\
\text { long }\end{array}$ & 13,153 & P06702 & $\begin{array}{l}\text { Acetyl, M missing } \\
\text { (N-term) }\end{array}$ \\
\hline $\begin{array}{l}\text { S100A9 (calgranulin) } \\
\text { long P }\end{array}$ & 13,233 & P06702 & $\begin{array}{l}\text { Acetyl, } M \text { missing } \\
\text { (N-term), phosph } \\
\left(T_{112}\right)\end{array}$ \\
\hline S100A12 (calgranulin C) & 10,444 & P80511 & M missing ( $\mathrm{N}$-term) \\
\hline
\end{tabular}

problems. A significant positive correlation was found for all the $\alpha$-defensins and S100A8 protein, whereas no correlation was found for S100A12 protein.

The results of the two-way ANOVA after rank transforming the values of the markers of inflammation confirmed a significant association between $\mathrm{Mn}$ and $\alpha$-defensin $1(P=0.05)$, $\alpha$-defensin $2(P=0.05), \alpha$-defensin $3(P=0.0091), \alpha$-defensin $4(P=0.0206)$, and $\mathrm{S} 100 \mathrm{~A} 12$ protein $(P=0.0125)$. The main effect of preterm premature rupture of membranes was not significant in any of the evaluations.

\section{DISCUSSION}

The aim of our study was to investigate whether the presence of Candida $\mathrm{Mn}$ antigen in BALF of premature infants is associated with an inflammatory response in their lungs. Inflammation is a central component of acute responses to infection, and neutrophils are important mediators of the acute innate immune response. The production of peptides and small proteins with microbicidal activity collectively called antimicrobial peptides is commonly considered to be a primitive mechanism of immunity.

The results of this study show that early (within the first $48 \mathrm{~h}$ of life) Mn detection in BALF of preterm newborns is correlated with an inflammatory state, as demonstrated by the significantly higher levels of $\alpha$-defensins $1-4$ and S100A12 protein in the Mn-positive group as compared with the Mn-negative group.

These data corroborate our previous results showing that $\alpha$-defensins 1-4 play an important role in the innate response to pulmonary infection in preterm newborns (21). Moreover, it has been already demonstrated that $\mathrm{S} 100 \mathrm{~A} 12$ protein has specific antifungal function, dependent on its $\mathrm{C}$-terminal peptide 


\section{Articles | Vento et al.}

a

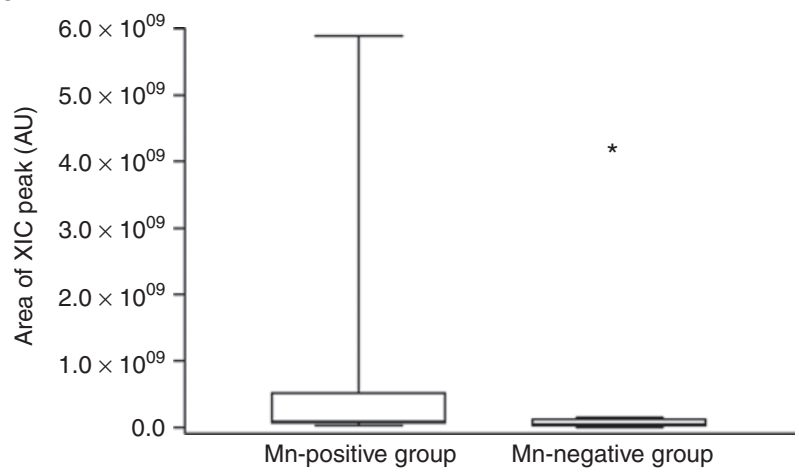

C

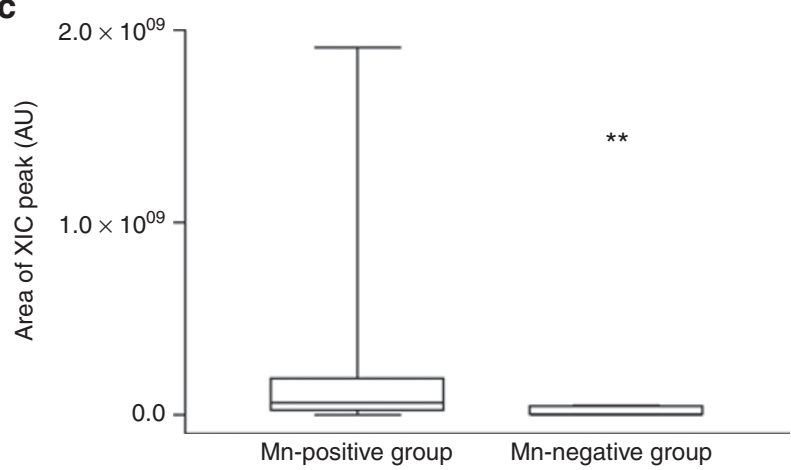

b

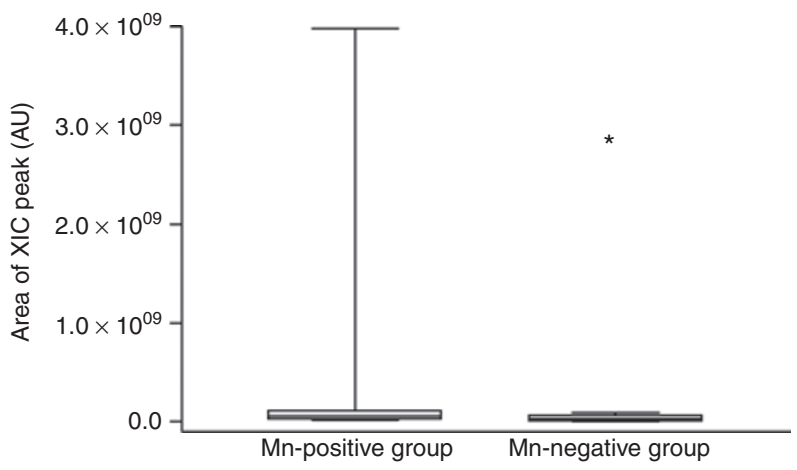

d

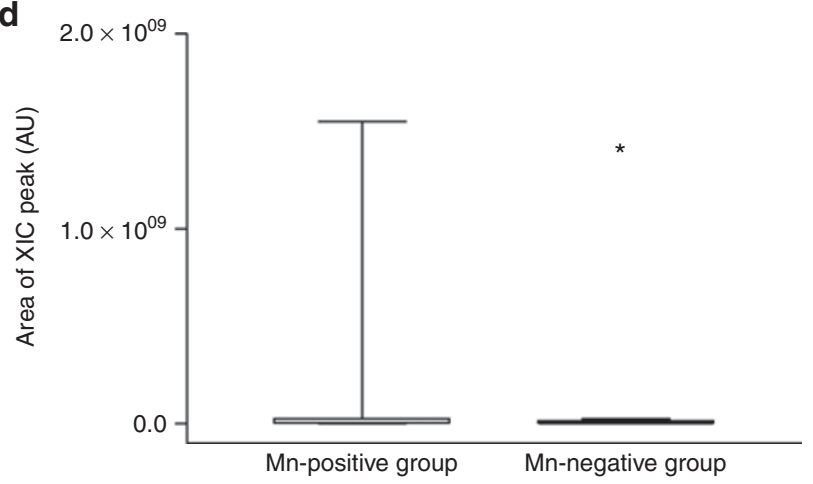

Figure 1. Individual and mean values of $\alpha$-defensins in the studied newborns. (a) Values of $\alpha$-defensin 1. (b) Values of $\alpha$-defensin 2. (c) Values of $\alpha$-defensin 3. (d) Values of $\alpha$-defensin $4 .{ }^{*} P=0.04 ;{ }^{* *} P=0.01$. AU, arbitrary units; XIC, extracted ion current.

a

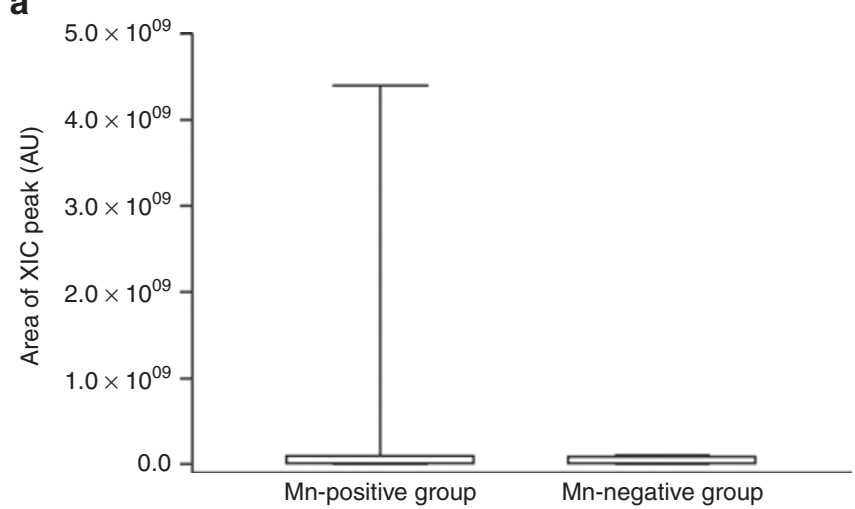

b

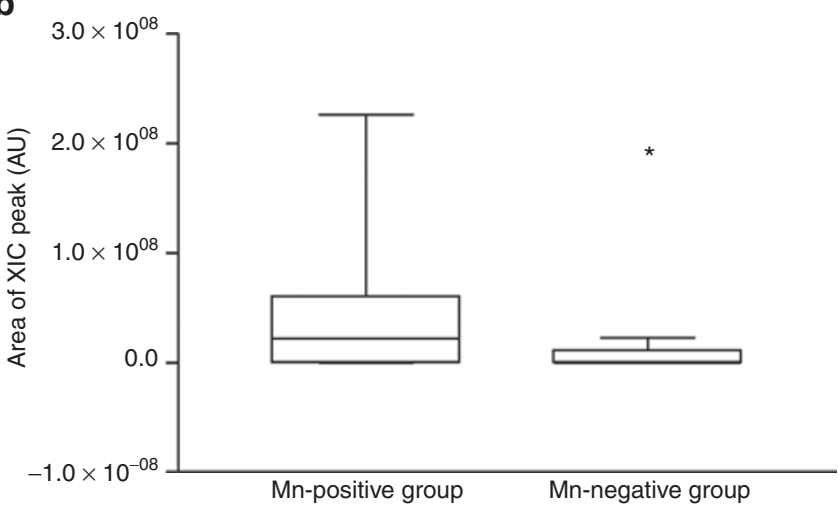

Figure 2. Individual and mean values of (a) S100A8 and (b) S100A 12 proteins in the studied newborns. ${ }^{*} P=0.03$. AU, arbitrary units; XIC, extracted ion current.
(26). To our knowledge, this is the first study suggesting that S100A12 could play a role in the innate response of preterm infants against Candida spp. lung infections.

The levels of the inflammatory markers may be influenced by maternal antifungal treatment or neonatal prophylaxis with fluconazole. Whereas no data are available concerning antifungal maternal therapy, all the studied neonates received a dose of fluconazole before the BALF collection. Furthermore, the antifungal prophylaxis, although potentially responsible for the levels of inflammatory markers, should not be a confounding factor in the analysis of the results of our study. The significant positive correlation between the absolute number of neutrophils and the corresponding levels of $\alpha$-defensins $1-4$ suggests that, even in preterm neonates, $\alpha$-defensins $1-4$ might originate prevalently by neutrophilic cells.

With regard to the absolute number of neutrophils in BALF, there were no significant differences between the two groups. However, the production of larger quantities of all the $\alpha$-defensins and S100A12 protein in the Mn-positive group, as compared with Mn-negative group, should testify that neutrophilic activation has occurred. We speculate that the neutrophilic activation and the production of antimicrobial peptides occur earlier than the recruitment of neutrophils. This is in accordance with the function of calgranulins and $\alpha$-defensins as chemoattractants, implicated in the neutrophil influx during infections $(15-17,27,28)$. In our previous study (21), a significantly higher absolute number of neutrophils was found in BALF samples of the pneumonia group than in the 
nonpneumonia group, but the BALF samples were collected in most cases on the 5th $\mathrm{d}$ of life, whereas the samples were all collected in this study within the first $48 \mathrm{~h}$ of life.

S100A8 and S100A9 proteins form the heterodimer calprotectin, with demonstrated antimicrobial activity against numerous microorganisms, particularly fungi $(26,29-32)$. However, when tested alone, neither S100A8 nor S100A9 showed activity in the Candida growth assay (31). In our study, none of the analyzed S100A9 isoforms were detected, except in two samples of the Mn-positive group. By contrast, S100A8 protein was detected in all the samples, but there were no significant differences between the two groups.

In all studied patients, BALF samples were obtained within the first $48 \mathrm{~h}$ of life. Therefore, the presence of Candida $\mathrm{Mn}$ antigen was observed very early in the Mn-positive group and bound with higher levels of some of the inflammatory markers that have been demonstrated to be associated with histological chorioamnionitis, funisitis, and early-onset neonatal sepsis $(25,33)$. It is possible to speculate a vertical transmission of Candida spp. infection. In fact, the presence of neutrophil defensins 1 and 2 and calgranulins $\mathrm{C}$ and $\mathrm{A}$ in the AF is highly predictive of histological chorioamnionitis, funisitis, and early-onset neonatal sepsis and seems to be the consequence of fetal neutrophilic activation. As demonstrated by Buhimschi et al. in the AF, S100A12 protein is strongly associated with funisitis, whereas S100A8 protein is linked with neonatal sepsis (a more advanced stage of disease). Authors suggest that there is a specific temporal sequence in the appearance of these biomarkers in women with intra-amniotic infection/inflammation because the S100A12 protein is produced in the early stage of fetal immune response to intra-amniotic infection. Therefore, S100A12 protein could be expressed by neutrophils in the first stages of acute immune response (25). In this sense, the finding of significantly higher levels of only S100A12 protein in our Mn-positive patients could reflect an early stage of fetal inflammation/infection, and it is plausible that Candida $\mathrm{Mn}$ antigen, as well as the cell wall $\beta$-glucan, induces the production of inflammatory bioproducts from phagocytes in the neonatal lung. However, the absence of detectable levels of S100A12 in most of our samples (13 of 22) could explain, at least in part, the lack of a significant correlation between the absolute neutrophil count and levels of S100A12 in our patients.

Different studies underscore the fact that antimicrobial peptides play a role in the inflammatory lung diseases and how, besides their host defense functions during infections, their proinflammatory activity is likely to have negative consequences $(15,27)$. As observed in the pneumonia group of our previous study (21), in the current study, a trend of higher incidence of bronchopulmonary dysplasia was observed in the Mn-positive group. Nevertheless, this study was not powered to detect differences in clinical outcomes such as bronchopulmonary dysplasia, and definitive conclusions cannot be made.

These results demonstrate an association between the $\mathrm{Mn}$ detection in BALF and the expression of $\alpha$-defensins 1-4 and S100A12 protein, as markers of inflammatory condition.
Therefore, Candida Mn detection in BALF can diagnose an early status of infection by Candida spp. in the respiratory tract of preterm newborns, and it is useful to actuate a preemptive treatment able to reduce ICI incidence and mortality in the neonatal intensive care unit (8).

\section{METHODS}

\section{Reagents and Instruments}

All general chemicals and reagents were of analytical grade and were purchased from Farmitalia-Carlo Erba (Milan, Italy), Merck (Damstadt, Germany), or Sigma-Aldrich (St. Louis, MO). The highperformance liquid chromatography (HPLC)-electrospray ionization (ESI)-mass spectrometer (MS) apparatus was a surveyor HPLC system connected by a $\mathrm{T}$ splitter to a Photo-diode Array detector (ThermoFinnigan, San Jose, CA) and to an Ion trap LCQ Deca XP Plus mass spectrometer (ThermoFinnigan). The chromatographic column was a Vydac (Hesperia, CA) C8 column, with 5- $\mu$ m particle diameter (column dimensions $150 \times 2.1 \mathrm{~mm}$ ).

\section{Human Participants}

This prospective observational study was performed in the neonatal intensive care unit of the Catholic University of Rome. Neonates with gestational age $\leq 27 \mathrm{wk}$ and/or birth weight $\leq 750 \mathrm{~g}$ consecutively admitted between January 2010 and June 2011 were enrolled, on the basis of our previous study results showing that newborns with the above-mentioned characteristics are at higher risk of ICI (34). They were eligible if they fulfilled the following criteria: (i) born in our hospital, (ii) endotracheal intubation was required at birth, and (iii) ongoing intensive care and mechanical ventilation were required. Newborns with major congenital malformations were excluded. Surfactant (a pig-derived natural surfactant, Curosurf; Chiesi Farmaceutici, Parma, Italy) was administered to all studied newborns, at a dose of $200 \mathrm{mg} / \mathrm{kg}$, as soon as possible after birth, always in the neonatal unit. All the babies were ventilated in elective high-frequency oscillatory ventilation modality with Draeger Babylog 8000 plus (Draeger, Lubeck, Germany) (35) and received ibuprofen therapy if the ductus arteriosus was hemodynamically significant (36).

According to our institutional protocol (35), all neonates with gestational age $\leq 27$ wk or birth weight $\leq 750 \mathrm{~g}$ received antifungal prophylaxis with fluconazole in the first $24 \mathrm{~h}$ of life and subsequently twice a week at the dosage of $3 \mathrm{mg} / \mathrm{kg} / \mathrm{d}$ intravenously (i.v.) (37). The antifungal prophylaxis was discontinued when no further need for intravenous access (central or peripheral) occurred, or when ICI developed. Likewise, when Mn antigen was detected in the BALF, the antifungal prophylaxis was stopped, and a treatment with liposomal amphotericin $\mathrm{B}$ was started at the dosage of $3 \mathrm{mg} / \mathrm{kg} / \mathrm{d}$ i.v. on the first day of therapy and subsequently a dosage of $5 \mathrm{mg} / \mathrm{kg} / \mathrm{d}$ i.v., for at least $14 \mathrm{~d}$.

The study protocol and consent forms were approved by the Ethics Committee of the Department of Pediatrics of the Catholic University of Rome, and the parents gave their informed consent. The incidence of some clinical outcomes was evaluated: ICI (defined as the presence of clinical signs of infection with a positive blood and/or urine and/or cerebrospinal fluid culture for fungi), bacterial sepsis (defined as the presence of clinical signs of infection with a positive blood culture for bacteria), bacterial pneumonia (defined as worsening of respiratory status with increased amounts of secretions by endotracheal tube, rales, wheezing, persistent chest radiographic abnormalities, and a positive BALF culture for bacteria), severe intracranial hemorrhage (grade III or IV), necrotizing enterocolitis (stage $>2$ ), ductus arteriosus surgically ligated, retinopathy of prematurity (stage $>2)$, bronchopulmonary dysplasia $\left(\mathrm{O}_{2}\right.$ dependence at $36 \mathrm{wk}$ of postmenstrual age), duration of mechanical ventilation, $\mathrm{O}_{2}$ therapy, and survival to discharge.

\section{BALF Collection and Processing}

BALF samples were obtained within the first $48 \mathrm{~h}$ of life according to a standardized procedure (35). Briefly, two sequential and separate aliquots of $1 \mathrm{ml} / \mathrm{kg}$ of $0.9 \%$ sodium chloride were instilled through a 
6-Fr gauge suction catheter that had been introduced via a slide valve at the endotracheal tube connection and advanced gently until resistance was met, immediately followed by suctioning. After collection, specimens were centrifuged at $1,000 \mathrm{~g}$ for $3 \mathrm{~min}$. Cells were suspended in $250 \mu \mathrm{l}$ of $0.9 \%$ sodium chloride, and the absolute and differential cell counts were obtained by automatic analyzer (Siemens-ADVIA 120; Hematology System, Tarrytown, NY) and by spinning $25 \mu \mathrm{l}$ of suspended cells onto two glass slides using May-Grunwald-Giemsa stain, as previously described (38). Trifluoroacetic acid $0.2 \%$ (vol/ vol) solution was immediately added to cell-free supernatants of BALF samples in 1:1 (vol/vol) ratio, and the solution was centrifuged at $8,000 \mathrm{~g}$ for $5 \mathrm{~min}$. The acidic treatment reduced the action of BALF proteases and artifact occurrence. After centrifugation, the acidic supernatants were separated from the precipitate, freeze-dried, lyophilized, and then resuspended in $300 \mu \mathrm{l}$ of $0.2 \%$ trifluoroacetic acid solution. One hundred microliters of this soluble fraction for each sample were analyzed by HPLC-ESI-MS.

\section{Candida Mn Antigen Detection}

BALF samples underwent Candida $\mathrm{Mn}$ antigen detection by the Platelia Candida Ag enzyme-linked immunosorbent assay immunoenzymatic sandwich microplate assay method (Bio-Rad Laboratories, Hercules, CA) according to the manufacturer's instructions. The assay uses the rat monoclonal antibody EB-CA1, which is directed against the Candida $\mathrm{Mn}$ molecule. Values of $\geq 0.25 \mathrm{ng} / \mathrm{ml}$ were considered positive for $\mathrm{Mn}$. This cutoff was suggested by the manufacturer. On the basis of the Mn test results, the studied newborns were divided in two groups: Mn-positive and Mn-negative groups. In addition, BALF samples were cultured for microbiological analysis including bacteria, Mycoplasma spp., Chlamydia, and fungi to diagnose lung infection.

\section{HPLC-ESI-MS Analysis}

The following solutions were used as mobile phases for the chromatographic separation in low-resolution ESI-MS mode: (eluent A) $0.056 \%$ aqueous trifluoroacetic acid and (eluent B) $0.050 \%$ trifluoroacetic acid in acetonitrile water $80 / 20$ ( $\mathrm{vol} / \mathrm{vol})$. The gradient applied was linear from 0 to $55 \%$ in $40 \mathrm{~min}$, at a flow rate of $0.30 \mathrm{ml} / \mathrm{min}$. The T splitter addressed a flow rate of $\sim 0.20 \mathrm{ml} / \mathrm{min}$ toward the diode array detector and $0.10 \mathrm{ml} / \mathrm{min}$ toward the ESI source. During the first 5 min of separation, the eluate was not addressed to the mass spectrometer to avoid instrument damage due to the high salt concentration. The Photo-diode Array detector was set at a wavelength of 214 and $276 \mathrm{~nm}$. Mass spectra were collected every $3 \mathrm{~ms}$ in the positive ion mode. MS spray voltage was $5.0 \mathrm{kV}$, and the capillary temperature was $250^{\circ} \mathrm{C}$.

\section{Relative Quantification of $\alpha$-Defensins and S100A Proteins}

$\alpha$-Defensins 1-4, S100A8, S100A9 (four isoforms), and S100A12 were previously detected, identified, and characterized in premature infants' saliva and BALF, from our group $(21,39)$. The relative abundance of $\alpha$-defensins $1-4$, S100A8, four isoforms of S100A9, and S100A12 proteins, among the different samples, was determined by considering the extracted ion current peak area. The extracted ion current procedure was based on the extraction of the current associated with three multiply charged ions specific for each $\alpha$-defensin and the proteins belonging to the S100A family from the total ion current chromatographic profile. It is now widely accepted that for the same protein and under constant experimental conditions, as those used in this study, the area of the extracted ion current peak is proportional to the amount and thus may be used for relative quantification (40). Indeed, we found a good linear correlation between the extracted ion current and ultraviolet peak areas of $\alpha$-defensins. Areas were not transformed in concentrations due to the lack of standards for all the peptides/proteins investigated. According to the European Respiratory Society Task Force recommendations for bronchoalveolar lavage in children (41), we analyzed and expressed the data without normalizing for dilution.

\section{Sample Size}

The study aims to open a new path in the early identification of patients at high risk of ICI by introducing new biomarkers. Phase I exploratory studies for biomarker development are mainly hypothesis generating, and formal sample size calculations are generally not needed (42). We used a convenience sample of 25 infants to explore fungi-driven inflammation.

\section{Data Treatment and Statistical Analysis}

Categorical variables were compared using a two-tailed Fisher's exact test. Testing for differences of continuous variables between groups was accomplished by Mann-Whitney $U$ test for nonparametric data and $t$-test for parametric data, as appropriate. To evaluate the relationship between $\alpha$-defensins, S100A proteins and absolute neutrophil counts in BALF samples, linear regression analysis was used. To take into account the role of the premature prolonged rupture of the fetal membranes when determining the association between the presence of $\mathrm{Mn}$ and the studied markers of inflammation, a two-way factorial ANOVA was performed after rank transforming the markers of inflammation values. The statistical software used included Instat (GraphPad PRISM Version 3.02; GraphPad Software, La Jolla, CA) and STATA 12 (Stata Corporation, College Station, TX). A $P$ value of $<0.05$ was considered statistically significant.

\section{Disclosure: The authors declared no conflict of interest.}

\section{REFERENCES}

1. Manzoni P, Farina D, Leonessa M, et al. Risk factors for progression to invasive fungal infection in preterm neonates with fungal colonization. Pediatrics 2006;118:2359-64.

2. Farmaki E, Evdoridou J, Pouliou T, et al. Fungal colonization in the neonatal intensive care unit: risk factors, drug susceptibility, and association with invasive fungal infections. Am J Perinatol 2007;24:127-35.

3. Rowen JL, Rench MA, Kozinetz CA, Adams JM Jr, Baker CJ. Endotracheal colonization with Candida enhances risk of systemic candidiasis in very low birth weight neonates. J Pediatr 1994;124(5 Pt 1):789-94.

4. Brian Smith P, Steinbach WJ, Benjamin DK Jr. Invasive Candida infections in the neonate. Drug Resist Updat 2005;8:147-62.

5. Brecht M, Clerihew L, McGuire W. Prevention and treatment of invasive fungal infection in very low birth weight infants. Arch Dis Child Fetal Neonatal Ed 2009;94:F65-9.

6. Pappas PG, Kauffman CA, Andes D, et al.; Infectious Diseases Society of America. Clinical practice guidelines for the management of candidiasis: 2009 update by the Infectious Diseases Society of America. Clin Infect Dis 2009;48:503-35.

7. Greenberg RG, Benjamin DK Jr, Gantz MG, et al.; Eunice Kennedy Shriver National Institute of Child Health and Human Development Neonatal Research Network. Empiric antifungal therapy and outcomes in extremely low birth weight infants with invasive candidiasis. J Pediatr 2012;161:264-9.e2.

8. Posteraro B, Sanguinetti M, Boccia S, et al. Early mannan detection in bronchoalveolar lavage fluid with preemptive treatment reduces the incidence of invasive Candida infections in preterm infants. Pediatr Infect Dis J 2010;29:844-8.

9. Gharib SA, Nguyen E, Altemeier WA, et al. Of mice and men: comparative proteomics of bronchoalveolar fluid. Eur Respir J 2010;35:1388-95.

10. Magi B, Bargagli E, Bini L, Rottoli P. Proteome analysis of bronchoalveolar lavage in lung diseases. Proteomics 2006;6:6354-69.

11. Wattiez R, Falmagne P. Proteomics of bronchoalveolar lavage fluid. J Chromatogr B Analyt Technol Biomed Life Sci 2005;815:169-78.

12. Noël-Georis I, Bernard A, Falmagne P, Wattiez R. Database of bronchoalveolar lavage fluid proteins. J Chromatogr B Analyt Technol Biomed Life Sci 2002; 771:221-36.

13. McMorran BJ, Patat SA, Carlin JB, et al. Novel neutrophil-derived proteins in bronchoalveolar lavage fluid indicate an exaggerated inflammatory response in pediatric cystic fibrosis patients. Clin Chem 2007;53:1782-91.

14. Lorenz E, Muhlebach MS, Tessier PA, et al. Different expression ratio of S100A8/A9 and S100A12 in acute and chronic lung diseases. Respir Med 2008;102:567-73.

15. Bals R. Epithelial antimicrobial peptides in host defense against infection. Respir Res 2000;1:141-50. 
16. Yoshio H, Lagercrantz H, Gudmundsson GH, Agerberth B. First line of defense in early human life. Semin Perinatol 2004;28:304-11.

17. Ganz T. Defensins and host defense. Science 1999;286:420-1.

18. Levy O. Antimicrobial proteins and peptides of blood: templates for novel antimicrobial agents. Blood 2000;96:2664-72.

19. Ashitani J, Mukae H, Hiratsuka T, Nakazato M, Kumamoto K, Matsukura S. Elevated levels of alpha-defensins in plasma and BAL fluid of patients with active pulmonary tuberculosis. Chest 2002;121:519-26.

20. Ashitani J, Mukae H, Hiratsuka T, Nakazato M, Kumamoto K, Matsukura S. Plasma and BAL fluid concentrations of antimicrobial peptides in patients with Mycobacterium avium-intracellulare infection. Chest 2001;119:1131-7.

21. Tirone $\mathrm{C}$, Boccacci S, Inzitari R, et al. Correlation of levels of alpha-defensins determined by HPLC-ESI-MS in bronchoalveolar lavage fluid with the diagnosis of pneumonia in premature neonates. Pediatr Res 2010;68:140-4.

22. Hsu K, Champaiboon C, Guenther BD, et al. Anti-infective protective properties of S100 calgranulins. Antiinflamm Antiallergy Agents Med Chem 2009;8:290-305.

23. Buhimschi IA, Christner R, Buhimschi CS. Proteomic biomarker analysis of amniotic fluid for identification of intra-amniotic inflammation. BJOG 2005;112:173-81.

24. Sampson JE, Theve RP, Blatman RN, et al. Fetal origin of amniotic fluid polymorphonuclear leukocytes. Am J Obstet Gynecol 1997;176(1 Pt 1):77-81.

25. Buhimschi CS, Buhimschi IA, Abdel-Razeq S, et al. Proteomic biomarkers of intra-amniotic inflammation: relationship with funisitis and early-onset sepsis in the premature neonate. Pediatr Res 2007;61:318-24.

26. Cole AM, Kim YH, Tahk S, et al. Calcitermin, a novel antimicrobial peptide isolated from human airway secretions. FEBS Lett 2001;504:5-10.

27. van Wetering S, Sterk PJ, Rabe KF, Hiemstra PS. Defensins: key players or bystanders in infection, injury, and repair in the lung? J Allergy Clin Immunol 1999;104:1131-8.

28. Koczulla AR, Bals R. Antimicrobial peptides: current status and therapeutic potential. Drugs 2003;63:389-406.

29. Steinbakk M, Naess-Andresen CF, Lingaas E, Dale I, Brandtzaeg P, Fagerhol MK. Antimicrobial actions of calcium binding leucocyte L1 protein, calprotectin. Lancet 1990;336:763-5.

30. Murthy AR, Lehrer RI, Harwig SS, Miyasaki KT. In vitro candidastatic properties of the human neutrophil calprotectin complex. J Immunol 1993;151:6291-301.
31. Sohnle PG, Hunter MJ, Hahn B, Chazin WJ. Zinc-reversible antimicrobial activity of recombinant calprotectin (migration inhibitory factor-related proteins 8 and 14). J Infect Dis 2000;182:1272-5.

32. Champaiboon C, Sappington KJ, Guenther BD, Ross KF, Herzberg MC. Calprotectin S100A9 calcium-binding loops I and II are essential for keratinocyte resistance to bacterial invasion. J Biol Chem 2009;284: 7078-90.

33. Buhimschi IA, Zambrano E, Pettker CM, et al. Using proteomic analysis of the human amniotic fluid to identify histologic chorioamnionitis. Obstet Gynecol 2008;111(2 Pt 1):403-12.

34. Vento G, Matassa PG, Ameglio F, et al. HFOV in premature neonates: effects on pulmonary mechanics and epithelial lining fluid cytokines. A randomized controlled trial. Intensive Care Med 2005;31: 463-70.

35. Su BH, Watanabe T, Shimizu M, Yanagisawa M. Echocardiographic assessment of patent ductus arteriosus shunt flow pattern in premature infants. Arch Dis Child Fetal Neonatal Ed 1997;77:F36-40.

36. Vendettuoli V, Tana M, Tirone C, et al. The role of Candida surveillance cultures for identification of a preterm subpopulation at highest risk for invasive fungal infection. Pediatr Infect Dis J 2008;27: 1114-6.

37. Kaufman DA. "Getting to zero": preventing invasive Candida infections and eliminating infection-related mortality and morbidity in extremely preterm infants. Early Hum Dev 2012;88:Suppl 2:S45-9.

38. Castagnola M, Inzitari R, Fanali C, et al. The surprising composition of the salivary proteome of preterm human newborn. Mol Cell Proteomics 2011;10:M110.003467.

39. Castagnola M, Cabras T, Iavarone F, et al. The human salivary proteome: a critical overview of the results obtained by different proteomic platforms. Expert Rev Proteomics 2012;9:33-46.

40. Vento G, Matassa PG, Zecca E, et al. Effect of dexamethasone on tracheobronchial aspirate fluid cytology and pulmonary mechanics in preterm infants. Pharmacology 2004;71:113-9.

41. de Blic J, Midulla F, Barbato A, et al. Bronchoalveolar lavage in children. ERS Task Force on bronchoalveolar lavage in children. European Respiratory Society. Eur Respir J 2000;15:217-31.

42. Pepe MS, Etzioni R, Feng Z, et al. Phases of biomarker development for early detection of cancer. J Natl Cancer Inst 2001;93:1054-61. 